\title{
UN MODELO DE CAJA APLICADO AL TRANSPORTE DE PARTICULAS Y TIEMPO DE RESIDENCIA DE LAS AGUAS DEL SECTOR "EL PINDO" (ENSENADA DE TUMACO)
}

\section{CCESP Jairo Javier Peña Gómez \\ Oceanógrafo Físico}

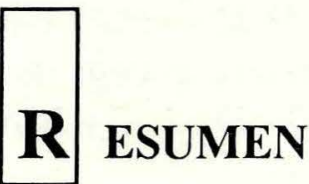

Este estudio se realizó con el fin de aplicar algunos principios de la Oceanografía Física para establecer las posibles causas de la persistencia de condiciones de baja calidad en las aguas del sector "El Pindo" en la Ensenada de Tumaco y proponer un mecanismo que potencialmente permita el restablecimiento de las condiciones físicas, químicas y biológicas.

Se realizaron jornadas de campo tendientes a determinar algunas características hidrológicas (temperatura, salinidad, sólidos suspendidos, oxígeno disuelto, mutrientes y transparencia), batimétricas, hidrodinámicas y meteorológicas del sector motivo de estudio. Los datos obtenidos fueron procesados estadísticamente y algunos de ellos considerados para la elaboración de un modelo de caja y en la consecuente determinación de los tiempos de residencia de estas aguas.

Básicamente, durante el periodo de estudio, se determinó que el sector se caracteriza por presentar bajas profundidades con extensos bancos de arena que en su desarrollo han ocasionado una reducción del canal navegable. La salinidad en marea alta presenta una marcada variación entre la superficie y el fondo, registrando valores entre $21.00 y$ $33.150 / 00$ 
en las aguas superficiales y 23.22 y 33.22 o/oo en las de fondo. La temperatura, presentó variaciones a lo largo de la columna de agua con un rango de variación de $23.20-29.52{ }^{\circ} \mathrm{C}$ y $26.88-29.18^{\circ} \mathrm{C}$ en fondo $y$ superficie, respectivamente.

Hidrodinámicamente se observó la presencia de un patrón de circulación muy particular, de acuerdo con el cual hay una evidente diferencia en el sentido de las corrientes durante cada mitad de los períodos de marea, el cual puede explicarse por los volúmenes de agua comprometidos. Puede concluirse que la calidad de las aguas del sector "El Pindo" no es consecuencia de este patrón de circulación sino que, fundamentalmente, depende de la calidad de los sedimentos de fondo lo cual podría subsanarse con un dragado de la zona.

Considerando el tiempo de residencia calculado para estas aguas (2-5 días), se recomienda el control en las actividades de los aserríos que se constituyen en una fuente de material que se deposita en el fondo, contribuyendo al deterioro de la calidad del agua y a la reducción de áreas profundas, con el consecuente efecto sobre las características químicas de los sedimentos. 


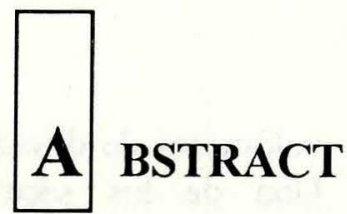

This study was carried out in order to apply several principles of the physical oceanography to determine the possible reasons for persistent low quality water conditions in "El Pindo" (Tumaco) area and propose a mechanism to recover physical, chemical and biological conditions.

The field work was done to determine different hydrological factors (temperature, salinity, suspended solids, dissolved oxygen, mutrients and transparency) as well as bathymetric, hydrodinamic and meteorological characteristics of the selected area. The obtained data were statistically processed and some of them were considered to outline a box model and consequently determine the residence time of these waters.

During the period of the study, it was found that shallow waters with wide sand banks causing the reduced shipway, are predominant. Salinity in high tide presents a remarkable variation between the surface and bottom values which were reported to be between $21.00-33.15$ o/oo and 23.22 - $33.22 \mathrm{o} / \mathrm{oo}$, respectively. Temperature variations along the water column were registered oscillating between $23.20-29.52{ }^{\circ} \mathrm{C}$ and 26.88 $29.18^{\circ} \mathrm{C}$ in botton and surface, respectively.

This area was found to have a particular hydrodinamic circulation pattern, existing an evident difference in the currents course in each tide period due to the involved water volumes. It is concluded that the low water quality of "El Pindo" area is not due to the circulation pattern but is caused by the bad bottom sediment quality and that could be improved by dredging to clean the waste area.

Considering the calculated residence times for these waters ( 2 - 5 days), it is recommended that control on sawmill activities should be carried out since these are the main source of material deposits in the bottom that affect the water quality and reduce the deep areas causing $a$ negative impact both on the predominant circulation pattern and chemical and biological characteristics of the bottom sediments. 


\section{NTRODUCCION}

Al considerar una masa de agua dada, generalmente, se espera encontrar características básicamente uniformes, las cuales se manifiestan en sus propiedades fisico-químicas en donde las mínimas variaciones, permiten formular conclusiones acerca del comportamiento general de las aguas comprometidas.

Las variaciones de la salinidad y temperatura entre aguas de superficie y fondo determinan una dinámica típica de cada sector. Tal es el caso de un estuario, donde el agua salada de mayor densidad, tiende a desplazarse por el fondo atribuyendo características especiales a este cuerpo de agua.

Un ejemplo de aguas estuarinas, lo ofrece la ensenada de Tumaco que recibe la influencia de varios ríos caudalosos, los cuales contribuyen a las variaciones de salinidad registradas en la zona.

Adicionalmente, -mantiene constante comunicación con el océano durante todo el año, con un fuerte componente mareal que produce notorias variaciones en su área. Esta ensenada ha presentado problemas de contaminación marina, atribuibles a la descarga de grandes volúmenes de desechos de impacto local. Uno de los sectores más seriamente afectados por procesos de contaminación es "El Pindo" donde, a los problemas de calidad de agua, se suman alteraciones hidrodinámicas ocasionadas, muy probablemente, por las modificaciones en la circulación como consecuencia de la construcción del puente de su mismo nombre. Las alteraciones en la calidad de estas aguas ha sido puesta de manifiesto en estudios previos (Marrugo, 1990; 1991 y Mosquera, 1992).

El flujo de agua en un punto y tiempo determinados, es el resultado de los efectos de la deriva del viento a través del tiempo, en combinación con las corrientes tanto permanentes como temporales, que resultan de los cambios de la distribución de las masas de agua. El océano está estratificado y sólo esporádicamente se vuelve turbulento en algunos lugares y las partículas suspendidas se mueven con poca mezcla a lo largo de superficies de densidad constante.

El transporte y difusión de una mancha de desechos, son propiedades modelables mediante el empleo de ecuaciones que contengan variables representativas que describan, en forma adecuada, el comportamiento de las propiedades involucradas. Es fundamental considerar la temperatura y salinidad en dichos modelos. 
El cambio de temperaturas en masas de agua, por debajo de la superficie, es una función del balance entre la advección y la difusión de energía produciéndose una conducción térmica de las aguas superficiales a las más profundas. Los datos necesarios para aplicar el modelo son una serie de determinaciones en diferentes puntos, desde la cabeza hasta la boca del estuario, bajo un rango de entrada de agua dulce y condiciones mareales, estimando una media diaria de ingreso de agua dulce.

Existen factores que determinan la tasa a la cual un cuerpo de agua intercambia nutrientes o contaminantes con el océano y ésta, a su vez, determina su estado trófico y de salud. El conocimiento de la tasa de transporte es importante para el manejo del mismo. Se han realizado varios estudios para estimar el ingreso de agua dulce y los tiempos de residencia en aguas con características estuarinas (Pritchard, 1960; Schauss, 1974; Conomos, 1979; Officcer, 1980; Takeoka, 1984; Pilson, 1985; Walters et. al., 1985; Goodwin, 1987; Miller y McPherson, 1991).

La importancia del presente estudio radica en que permite la aplicación de un modelo de caja para estimar el tiempo de residencia para el sistema en estudio y, con base en éste, propone un mecanismo de restauración de la dinámica para facilitar una rápida renovación de masas de agua y, por tanto, contribuye a restablecer las condiciones normales en el área, previniendo la extensión del estado eutrófico local a otros sectores de la ensenada.

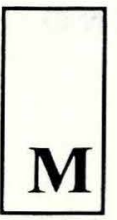

ATERIALES Y METODOS

\section{Area de Estudio}

La ensenada de Tumaco se halla localizada al suroccidente de Colombia en el Departamento de Nariño. Está delimitada entre latitudes $1^{\circ} \quad 45^{\prime}$ y $2^{\circ} \quad 00 \mathrm{~N}$ y longitudes $78^{\circ} 30^{\prime}$ y $78^{\circ} 45^{\prime} \mathrm{W}$. Presenta una forma alargada de Norte a Sur, con un área aproximada de $350 \mathrm{Km}$ (Figura 1).

El régimen mareal es semidiurno con una amplitud máxima de $4 \mathrm{~m}$. La profundidad de sus aguas varía entre 2 - $30 \mathrm{~m}$. Correa et. al., 1988, señalan que la mayor parte de la linea de costa de la ensenada, muestra evidencias de cambios morfológicos asociados a eventos de erosión y acrecimiento litoral presentes ocurridos en los últimos 30 - 40 años. En el fondo predominan fango y materia orgánica en descomposición y arenas que, junto con la afluencia de materiales transportados por los ríos, le dan a estas aguas una alta productividad y baja transparencia con elevados índices de sedimentación. 


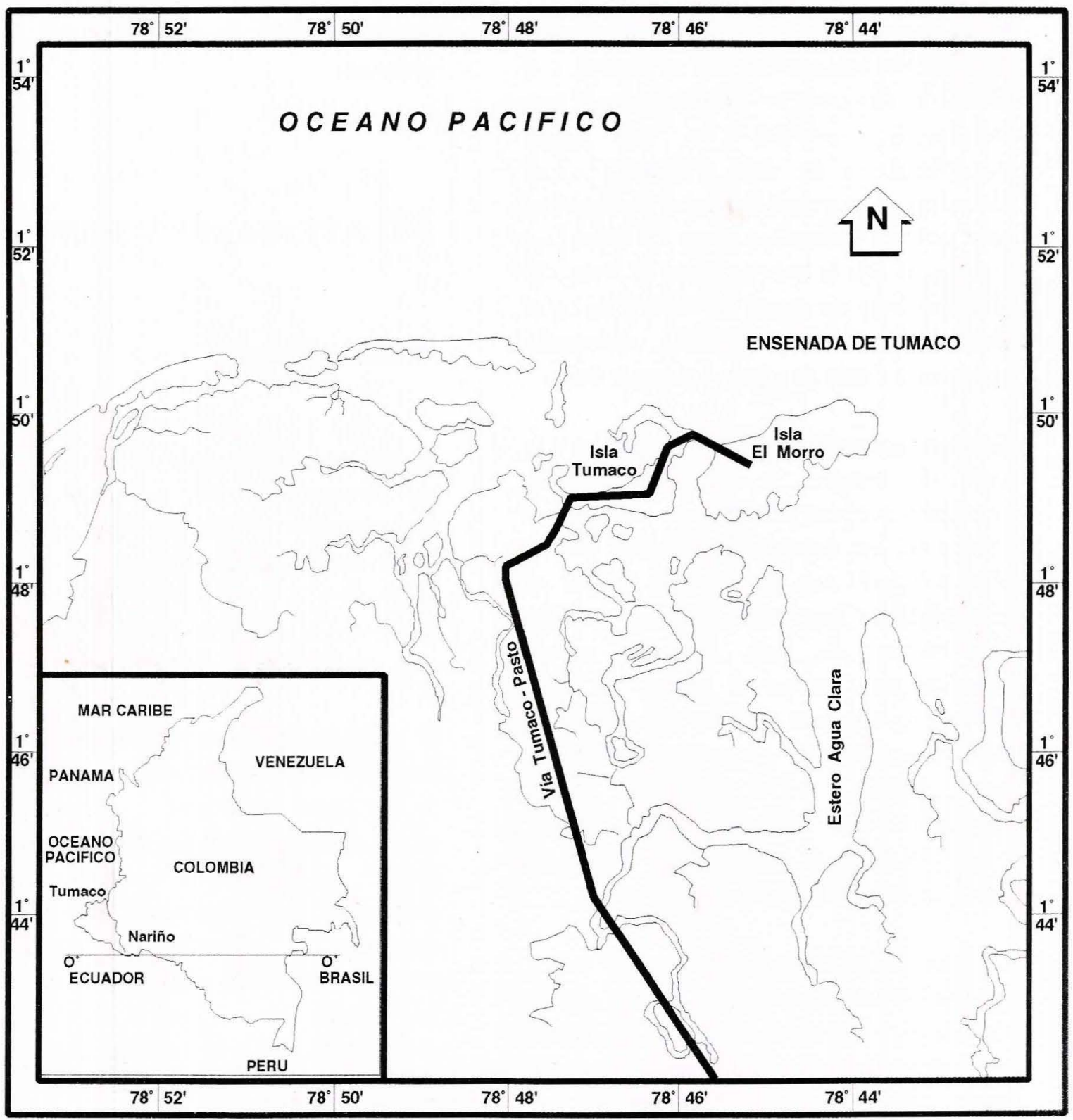

Figura 1

Ubicación del área de estudio 
Las temperaturas del aire son típicas de las zonas ecuatoriales. La máxima promedio, obtenida durante 1992 , alcanzó $29.62^{\circ} \mathrm{C}$ en abril; mientras que, la mínima promedio registrada en el mes de diciembre fue de $23.60{ }^{\circ} \mathrm{C}$. La humedad relativa promedio, para el mismo año, es $83.86 \%$ y registra un promedio de $3872.00 \mathrm{~mm}$ de precipitación anual, con un aumento de lluvias a lo largo del año, muy probablemente, debido al fenómeno $\mathrm{El}$ Niño manifestado en la costa pacífica durante 1992 .

La Isla de Tumaco está conectada a tierra firme por un puente corto denominado "El Pindo" (figura 2), que divide el área de estudio en dos sectores (occidental y oriental), y por $12 \mathrm{Km}$ de carretera a través de esteros, lagunas y, en general, zonas de bajamar cubiertas por mangle.

El Pindo, es uno de los sectores más afectados por la contaminación dentro de la Ensenada. En marea baja presenta el olor característico de la liberación de ácido sulfhídrico. Es común la presencia de alevinos muertos o en estado agonizante con movimientos descoordinados sobre la superficie del agua, claro síntoma de anoxia (Mosquera, 1992).

Esto puede explicarse porque, además de la escasa circulación de las aguas aledañas al sector, se ubican viviendas, aserraderos, el matadero municipal y sitios de venta de pescado que vierten sus subproductos (aserrín, vísceras, líquidos y demás) al mar. Todas estas condiciones parecen estar incidiendo en el desarrollo de un proceso paulatino de eutroficación en el área.

\section{Desarrollo de la investigación}

La metodología aplicada se fundamenta en la ejecución de jornadas diarias de campo en un período de dos meses (noviembre/92- diciembre/92), haciendo el seguimiento de los parámetros estudiados a lo largo de un ciclo completo de marea, a bordo de una lancha tipo taxi, anclada en cada sitio de muestreo. Las estaciones fueron referenciadas por los nodos de una red de cuadrículas separadas por una distancia de aproximadamente $40 \mathrm{~m}$.

\section{Características del cuerpo de agua}

Para la determinación de la salinidad y temperatura se tomaron registros de superficie y fondo en marea alta, utilizando un termosalinómetro portátil Beckman. No se obtuvieron datos en marea baja por considerarse ampliamente reducido el espejo de agua del sector. Los sólidos suspendidos fueron analizados en el laboratorio, aplicando el método descrito por APHA (1981), utilizando filtros de celulosa. El oxígeno disuelto se determinó 


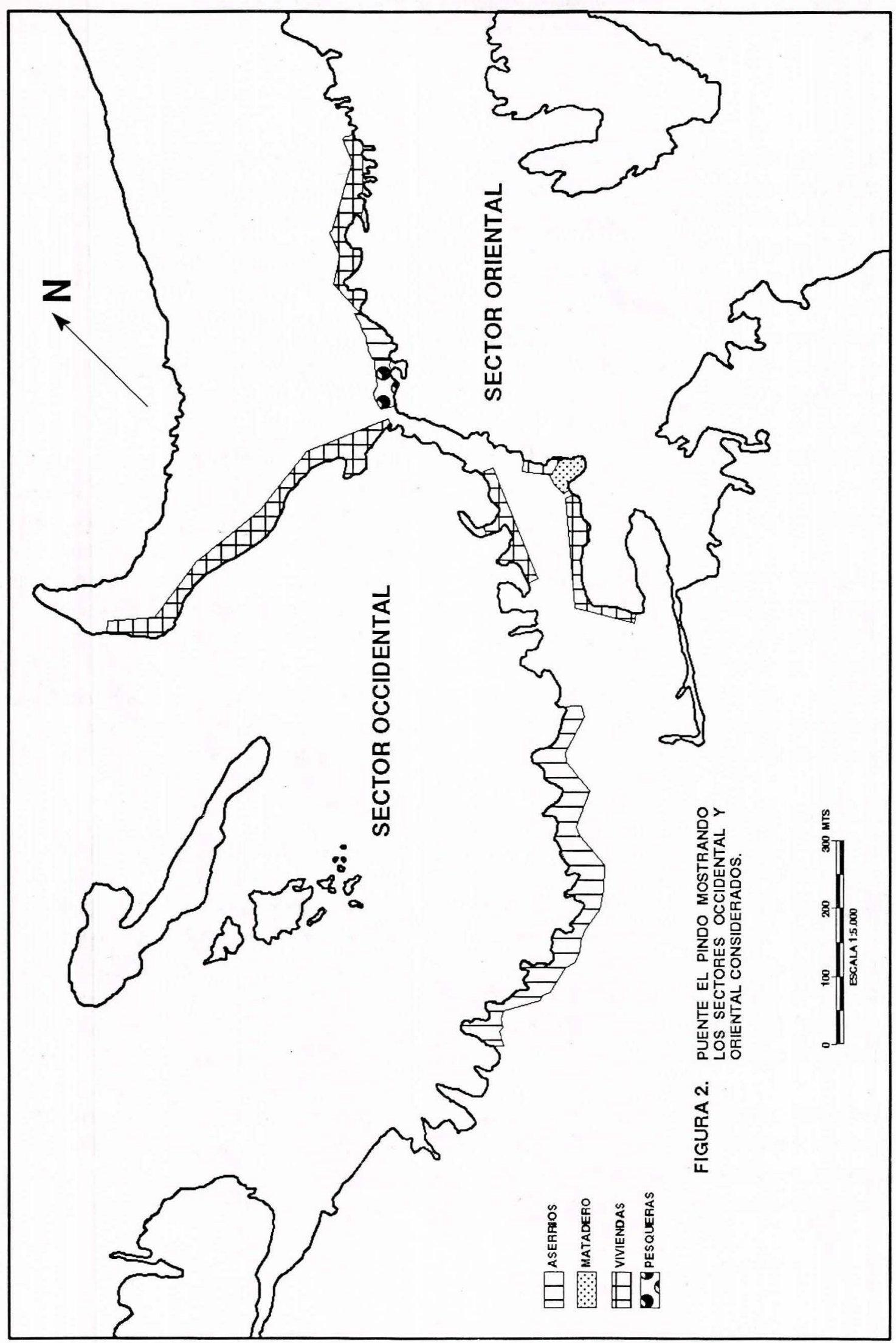


por el metodo Winkler y los nutrientes (nitritos;' nitratos y fosfatos) con el método colorimétrico de Strickland y Parsons (1972). La transparencia fue medida con el disco Secchi.

Para la caracterización del fondo en este sector, se utilizó una ecosonda de registro continuo en línea recta Sitex. Una vez obtenidos los perfiles batimétricos en los ecogramas, fueron trasladados a una carta a escala del sector estudiado.

Para la determinación de la velocidad y dirección de las corrientes, se aplicaron los métodos Lagrangiano y Euleriano. Los registros fueron obtenidos con un correntómetro digital marca Kahlsico.

Los datos meteorológicos (humedad relativa, temperatura del aire, evaporación, precipitación y dirección del viento) reportados, fueron obtenidos de la estación meteorológica del IDEAM en Tumaco.

\section{Modelo de caja y tiempos de residencia}

El modelo de caja aplicado en el presente trabajo se basó en el propuesto por Enfield (1976) y utilizado por Aguilera (1986).

De acuerdo con este modelo, las características de un cuerpo de agua se analizan en términos de entradas y salidas de componentes fisicos (masa, sal, sol, etc.) y sus respectivas intensidades (salinidad, temperatura, etc.). Acorde con lo expresado por Miller y McPherson (1991), el modelo se fundamenta en el hecho que sobre un período de varios ciclos mareales, el flujo mareal promedio de agua nueva con salinidad mayor o igual a $33.5 \%$ oo proveniente de aguas internas puede ser tratado como una constante en el área de estudio. Este flujo puede ser usado para predecir la salinidad en el estuario frente a un determinado aporte del río. El flujo de intercambio mareal y el aporte del río pueden usarse para mover material y para transportarlo desde el estuario.

\section{Características Hidrológicas}

\section{Parámetros fisicoquímicos generales}

La tabla 1, presenta los promedios reportados durante dos años de observación para transparencia, temperatura, salinidad, sólidos suspendidos, oxígeno disuelto, $\mathrm{DBO} 5$, nitrógeno total inorgánico ( $\mathrm{NO}_{2}+\mathrm{NO}_{3}+\mathrm{NH}_{4}$ ) fosfatos y clorofila en un sitio de muestreo fijo en el sector El Pindo; 


\begin{tabular}{|c|c|c|c|c|c|c|c|c|c|c|c|c|}
\hline \multicolumn{13}{|c|}{ OS $\triangle 1 O N E S$} \\
\hline \multirow[t]{2}{*}{ P/R AME HRO } & \multicolumn{6}{|c|}{ MARE A L LI } & \multicolumn{6}{|c|}{ MARE $/ \mathrm{B} / \mathrm{K}$} \\
\hline & \multicolumn{3}{|c|}{$\mathrm{ES} \cup 1 \mathrm{NDO}$} & \multicolumn{3}{|c|}{ WLPINDO } & \multicolumn{3}{|c|}{$\mathrm{HI} \vee \mathrm{YUDO}$} & \multicolumn{3}{|c|}{ KLIPINDO } \\
\hline MNASM $M$ MGK & $\begin{array}{l}4.11 \\
6.00 \\
\end{array}$ & $+/-$ & $\begin{array}{l}0.88 \\
2.70\end{array}$ & $\begin{array}{l}1.79 \\
2.70\end{array}$ & $+/-$ & $\begin{array}{l}0.38 \\
1.49 \\
\end{array}$ & $\begin{array}{l}2.90 \\
4.30 \\
\end{array}$ & $\begin{array}{r}+1 \\
- \\
\end{array}$ & $\begin{array}{l}1.05 \\
2.00 \\
\end{array}$ & $\begin{array}{l}0.89 \\
2.20 \\
\end{array}$ & $+/-$ & $\begin{array}{l}0.49 \\
0.40 \\
\end{array}$ \\
\hline M & $\begin{array}{l}27.49 \\
29.29 \\
\end{array}$ & $+/-$ & $\begin{array}{r}0.84 \\
27.00 \\
\end{array}$ & $\begin{array}{l}27.84 \\
30.16 \\
\end{array}$ & $+1-$ & $\begin{array}{r}1.18 \\
26.30 \\
\end{array}$ & $\begin{array}{l}27.92 \\
29.10\end{array}$ & $\begin{array}{r}+1 \\
-\end{array}$ & $\begin{array}{r}0.91 \\
27.00\end{array}$ & $\begin{array}{l}29.45 \\
31.50\end{array}$ & $+1-$ & $\begin{array}{r}0.93 \\
27.50\end{array}$ \\
\hline SY UIVIY & $\begin{array}{l}32.07 \\
32.50 \\
\end{array}$ & $+1-$ & $\begin{array}{r}0.40 \\
31.00 \\
\end{array}$ & $\begin{array}{l}28.84 \\
30.50 \\
\end{array}$ & $+/-$ & $\begin{array}{r}2.68 \\
24.60 \\
\end{array}$ & $\begin{array}{l}31.17 \\
31.50 \\
\end{array}$ & $\begin{array}{r}+1 \\
-\end{array}$ & $\begin{array}{r}0.89 \\
30.10 \\
\end{array}$ & $\begin{array}{l}29.86 \\
31.30 \\
\end{array}$ & $+1-$ & $\begin{array}{r}1.35 \\
29.60 \\
\end{array}$ \\
\hline SOWSUS & $\begin{array}{r}64.45 \\
109.00\end{array}$ & $+/-$ & $\begin{array}{l}20.65 \\
35.00\end{array}$ & $\begin{array}{r}68.09 \\
110.00\end{array}$ & $+/-$ & $\begin{array}{l}21.89 \\
34.00\end{array}$ & $\begin{array}{r}60.58 \\
100.00\end{array}$ & +1 & $\begin{array}{l}23.43 \\
21.00\end{array}$ & $\begin{array}{r}72.75 \\
133.00\end{array}$ & $+1-$ & $\begin{array}{l}30.01 \\
35.00\end{array}$ \\
\hline OXG OH & $\begin{array}{l}4.49 \\
5.03 \\
\end{array}$ & $+1-$ & $\begin{array}{l}0.32 \\
4.12 \\
\end{array}$ & $\begin{array}{l}3.55 \\
4.02 \\
\end{array}$ & $+1-$ & $\begin{array}{l}0.24 \\
3.20 \\
\end{array}$ & $\begin{array}{l}4.59 \\
4.97 \\
\end{array}$ & $\begin{array}{r}+1 \\
-\end{array}$ & $\begin{array}{l}0.35 \\
3.81 \\
\end{array}$ & $\begin{array}{l}3.16 \\
4.47 \\
\end{array}$ & $+1-$ & $\begin{array}{l}0.87 \\
1.62 \\
\end{array}$ \\
\hline 1010 & $\begin{array}{l}2.80 \\
5.30 \\
\end{array}$ & $+1-$ & $\begin{array}{l}2.63 \\
1.16 \\
\end{array}$ & $\begin{array}{r}8.23 \\
10.92 \\
\end{array}$ & $+1-$ & $\begin{array}{l}2.47 \\
4.02 \\
\end{array}$ & $\begin{array}{l}4.32 \\
8.00 \\
\end{array}$ & +1 & $\begin{array}{l}2.63 \\
1.08 \\
\end{array}$ & $\begin{array}{l}6.85 \\
7.25 \\
\end{array}$ & $+1-$ & $\begin{array}{l}0.48 \\
6.33 \\
\end{array}$ \\
\hline 1111 & $\begin{array}{l}1.75 \\
2.54\end{array}$ & $+/-$ & $\begin{array}{l}0.57 \\
0.75\end{array}$ & $\begin{array}{l}2.09 \\
2.70\end{array}$ & $+1-$ & $\begin{array}{l}0.42 \\
1.30\end{array}$ & $\begin{array}{l}1.68 \\
3.35 \\
\end{array}$ & +1 & $\begin{array}{l}0.87 \\
0.45 \\
\end{array}$ & $\begin{array}{l}.90 \\
4.22 \\
\end{array}$ & $+1-$ & $\begin{array}{l}0.98 \\
1.72\end{array}$ \\
\hline $1 \%$ T. & $\begin{array}{l}0.50 \\
0.88 \\
\end{array}$ & $+1-$ & $\begin{array}{l}0.21 \\
0.20 \\
\end{array}$ & $\begin{array}{l}0.69 \\
0.88\end{array}$ & $+/-$ & $\begin{array}{l}0.19 \\
0.34\end{array}$ & $\begin{array}{l}0.52 \\
0.93\end{array}$ & +1 & $\begin{array}{l}0.26 \\
0.22 \\
\end{array}$ & $\begin{array}{l}0.97 \\
1.60 \\
\end{array}$ & $+1-$ & $\begin{array}{l}0.35 \\
0.68 \\
\end{array}$ \\
\hline $100 \%$ & $\begin{array}{l}1.10 \\
2.09\end{array}$ & $+/-$ & $\begin{array}{l}1.09 \\
\text { N.D }\end{array}$ & $\begin{array}{l}1.53 \\
3.20 \\
\end{array}$ & $+/-$ & $\begin{array}{l}0.08 \\
\text { N.D }\end{array}$ & $\begin{array}{l}0.78 \\
1.76 \\
\end{array}$ & +1 & $\begin{array}{l}0.44 \\
0.21 \\
\end{array}$ & $\begin{array}{l}2.58 \\
4.45 \\
\end{array}$ & $+1-$ & $\begin{array}{l}1.13 \\
0.46 \\
\end{array}$ \\
\hline
\end{tabular}

* Nitrogeno total inorgánico $=\mathrm{NO}_{2}+\mathrm{NO}_{3}+\mathrm{NH}_{4}$

Interprétese como: promedio +/- Des. Stan.

Maximo Minimo

\section{Tabla 1}

Valores promedio y desviación estándar, de los parámetros fisicoquímicos, con registros máximos y mínimos reportados en las estaciones El Viudo y El Pindo (ensenada de Tumaco)

comparándolos con los registrados en la estación El Viudo que, para el caso de la ensenada de Tumaco, representa mejores condiciones fisicoquímicas por estar menos influenciada por agentes antropogénicos que actúan hacia el interior de la ensenada, con mayor incidencia en El Pindo.

De la tabla anterior, también se deduce claramente que existe una significativa diferencia para todos los parámetros entre los períodos de alta y baja marea, siendo evidente la disminución de oxígeno disuelto y transparencia y el aumento de DBO5, clorofila y nutrientes en estas estaciones, con una situación más crítica en El Pindo. Para el caso de la DBO5, se presenta un promedio aparentemente menor en marea baja con respecto a la alta; sin embargo, las desviaciones 
Un modelo de caja aplicado al transporte de partículas y tiempo de residencia de las aguas del sector "EL PINDO", Ensenada de Tumaco

estándar y los valores máximo y mínimo correspondientes, nos muestran que la situación se vuelve realmente más crítica en el sector El Pindo durante el período de baja marea.

\section{Salinidad}

Los valores de salinidad registrados durante el período de estudio para el sector El Pindo tanto en superficie como en el fondo, muestran que existe una mayor estratificación en el sector occidental del puente, donde es apreciable la presencia de una masa de agua superficial con valores entre $21.00-28.90$ o/oo y otra inferior variable entre 23.22 y 33.56 o/oo. En el sector oriental, por el contrario, se observa una masa de agua poco estratificada con valores superficiales en el rango $29.40-33.15$ o/oo y en el fondo, 29.60 - 33.22 o/oo. Considerando la distribución longitudinal (figuras 3 y 4), puede observarse que existe una tendencia al aumento de la misma superficialmente desde el área de ingreso de aporte fluvial hacia el puente, a través del canal central, con los máximos valores de salinidad superficial en las áreas circundantes a este sector. En los extremos del sector occidental se observan mayores valores de salinidad con una tendencia a disminuir en cercanías del puente. En el sector oriental existen menores variaciones de la salinidad superficial, sin mostrar una clara tendencia en la distribución longitudinal. Contrario a lo esperado, no existe mucha similitud entre los valores registrados para las estaciones contiguas, separadas por el puente. En el fondo no se observa una tendencia claramente definida; aunque en el sector occidental, en general, puede notarse un registro de valores menores en proximidad del puente con algunas áreas aisladas, con valores altos y bajos de salinidad a lo largo. En el sector oriental nuevamente se observan las menores variaciones sin una evidente tendencia de distribución longitudinal de los valores de la salinidad de fondo. En general, los registros salinos muestran una tendencia al aumento, en función directa de su distancia del aporte fluvial, con diferencias verticales muy importantes dependientes de la descarga de agua dulce que contribuye a disminuir la salinidad superficial.

\section{Temperatura}

En la parte occidental del puente El Pindo, la temperatura superficial varía entre 23.20 - $29.52{ }^{\circ} \mathrm{C}$, mostrando una tendencia muy poco definida. Sin embargo, con base en la distribución longitudinal, pueden esta-blecerse dos zonas desde el área de aporte fluvial hacia el puente, a través del canal central: la primera con una temperatura de 27.80

${ }^{C} C$ y otra con una temperatura de 27.70 ${ }^{\circ} \mathrm{C}$ separadas por un área con valor de $28.0^{\circ} \mathrm{C}$. Hacia los extremos, en general, la temperatura es menor con tendencia al aumento hacia el puente (Figura5). 


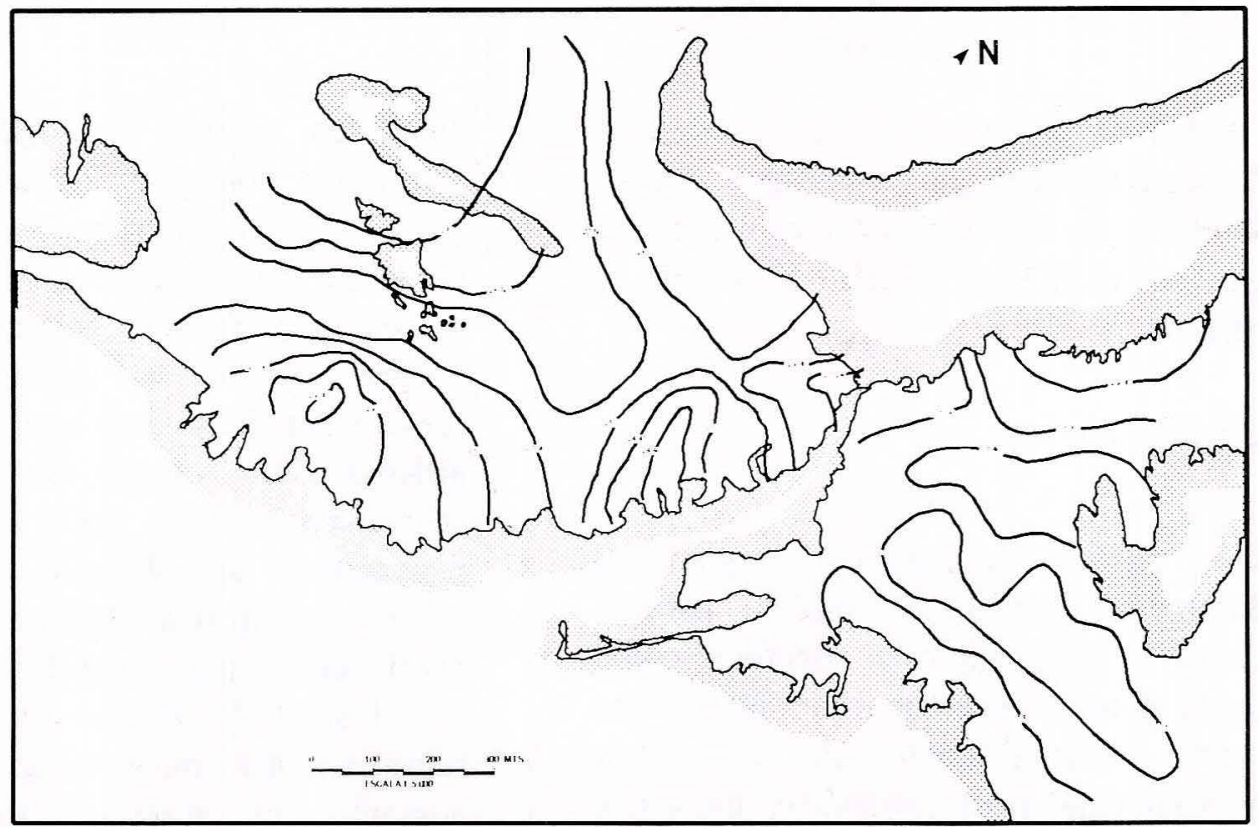

Figura 3

Distribución longitudinal de la salinidad superficial en el área de estudio

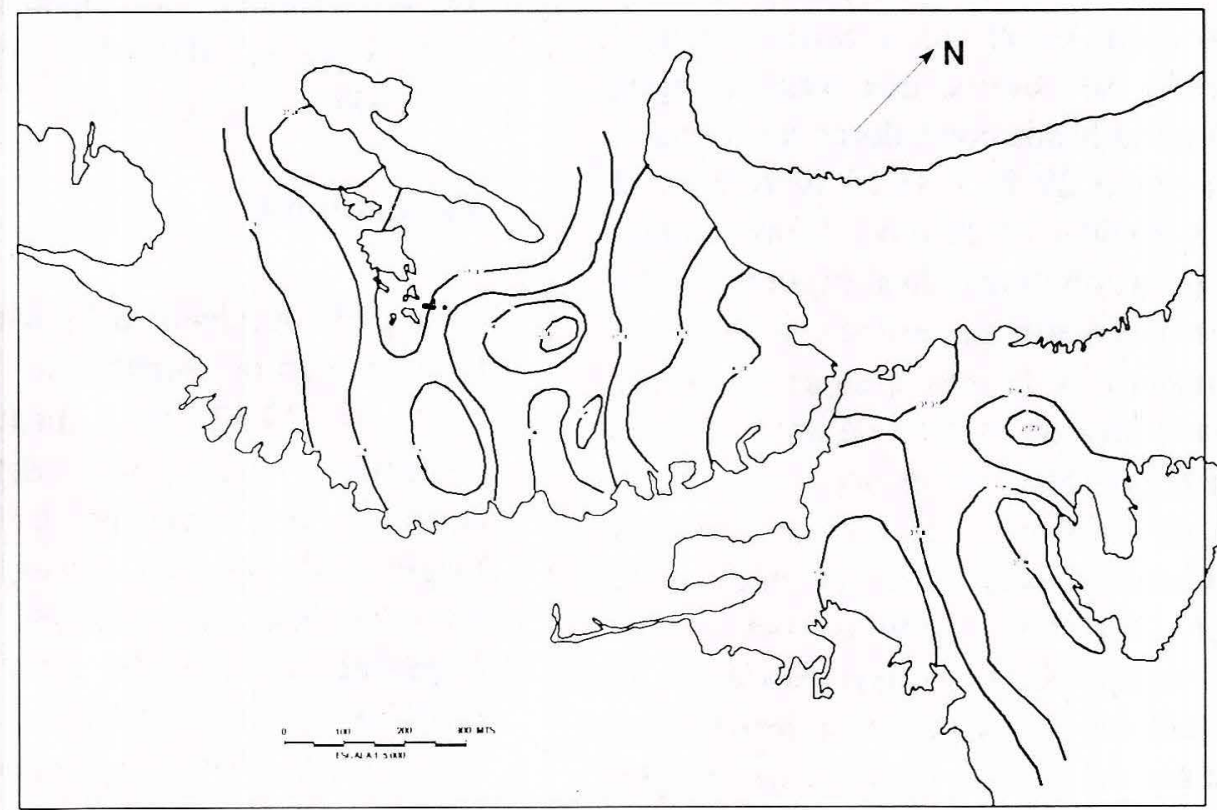

Figura 4

Distribución longitudinal de la salinidad de fondo del área de estudio 


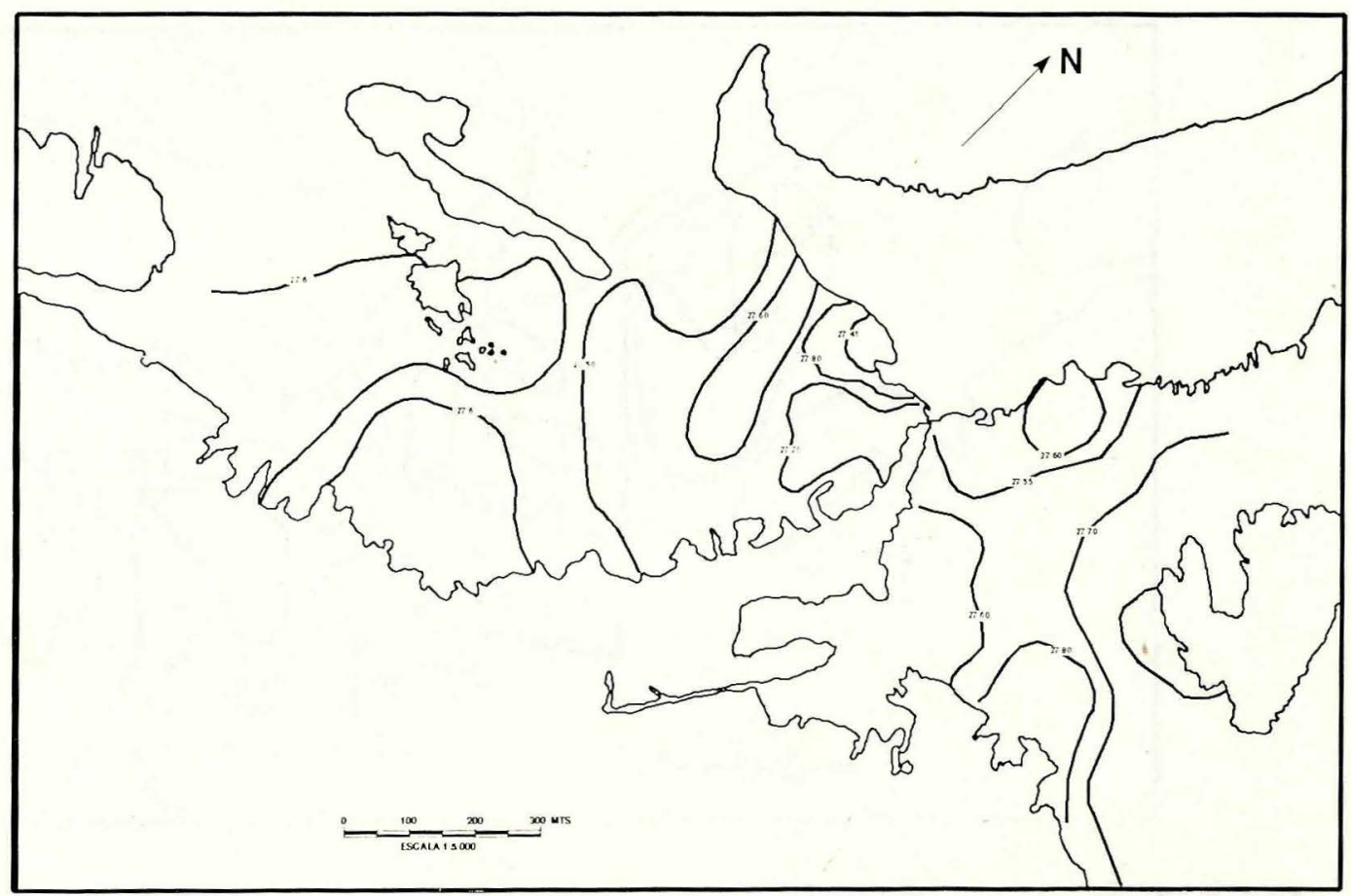

\section{Figura 5}

Distribución longitudinal de la temperatura superficial en el área de estudio

Del lado opuesto se observa una tendencia al aumento desde el puente, con los mayores valores en el extremo sudeste de este sector. Verticalmente no se observa una estratificación. Los valores de fondo variaron entre $26.88-29.18{ }^{\circ} \mathrm{C}$ con un comportamiento longitudinal muy similar al descrito para los registros en superficie (figura 6).

\section{Características Batimétricas.}

La figura 7, muestra las características predominantes en el relieve de fondo en el área de estudio. Básicamente, es una zona de aguas poco profundas, cuyas mayores profundidades se registran en el sector central, pero presenta una distribución batimétrica muy irregular. En el lado occidental del puente, se presentan las menores profundidades cerca a la costa y en el área circundante a la del ingreso fluvial. En el lado oriental, las menores profundidades se registran, igualmente, en el sector central con los menores valores cerca de la costa y en proximidad al bajo en desarrollo en medio del área de estudio. 


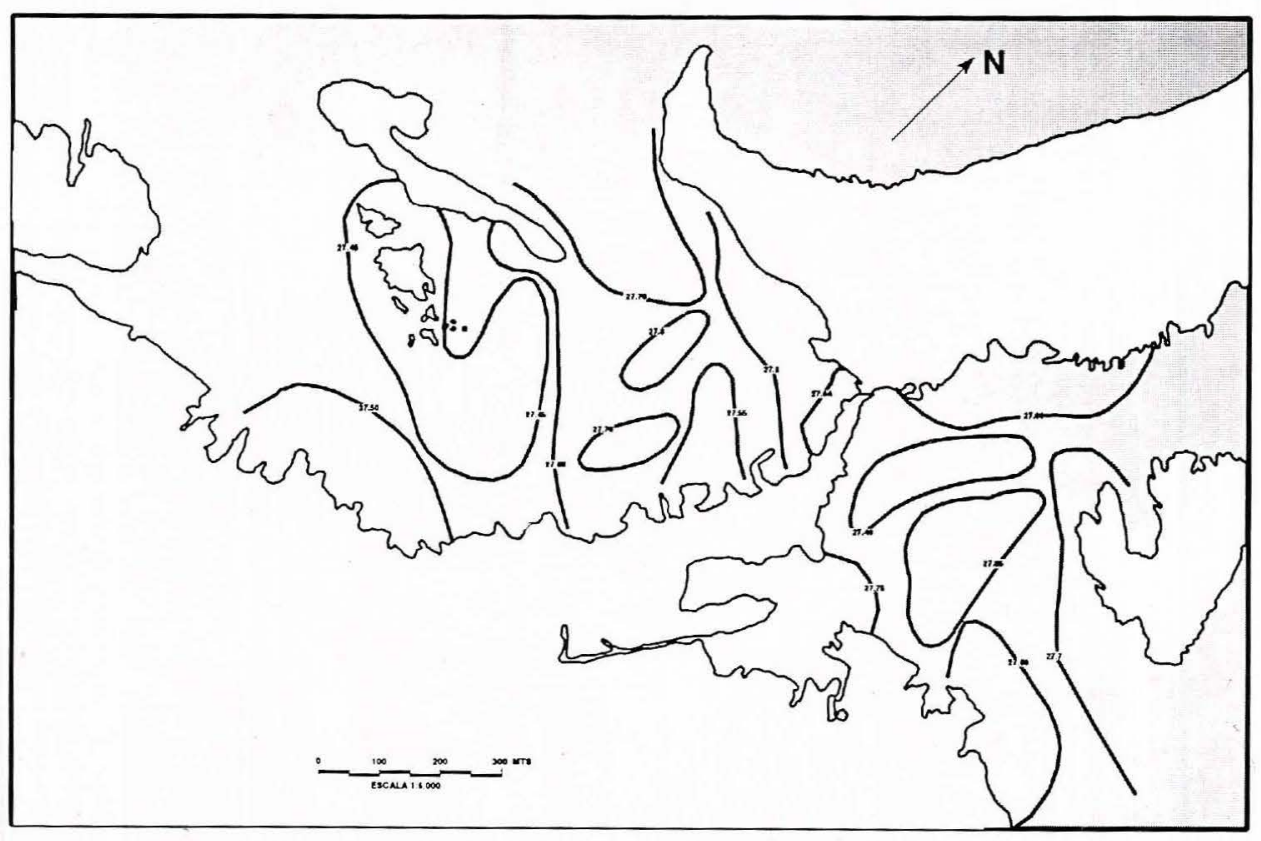

Figura 6

Distribución longitudinal de la temperatura del fondo en el área de estudio

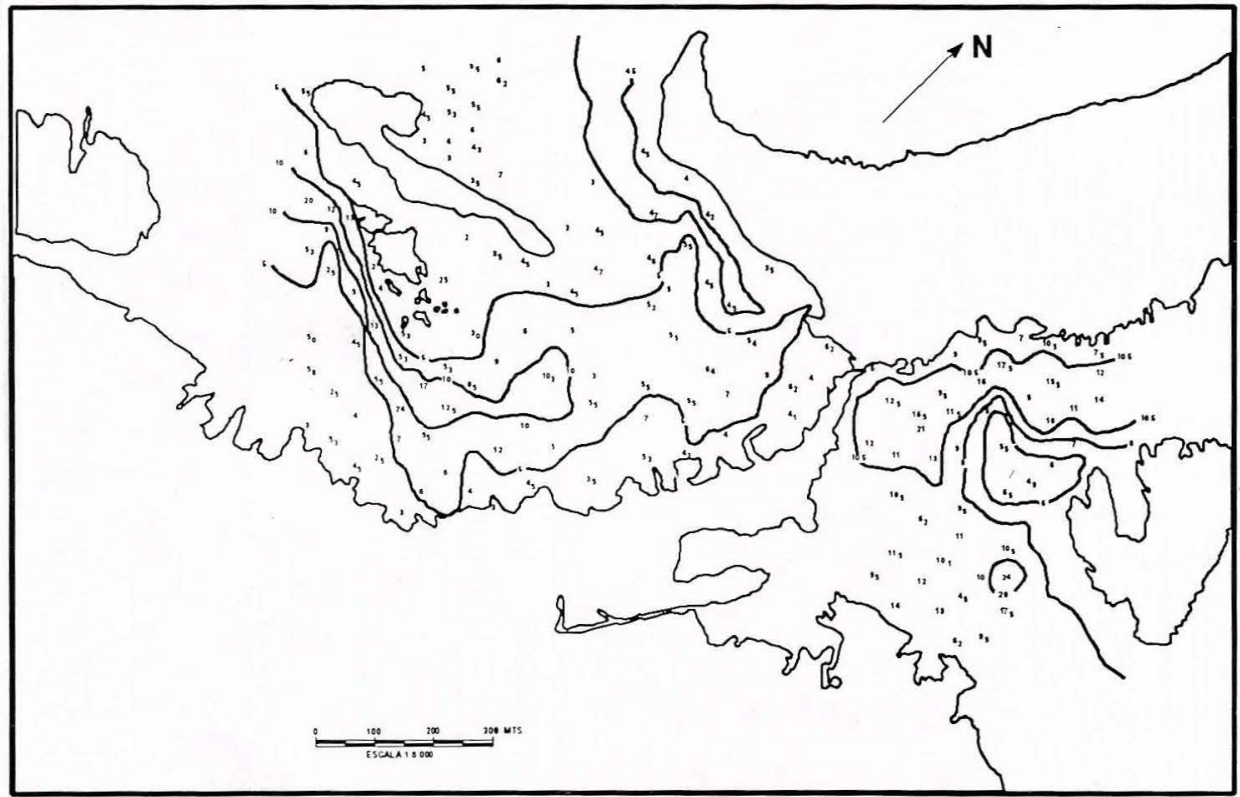

Figira 7

Características predominantes en el relieve de fondo en el área de estudio 
Un modelo de caja aplicado al transporte de particulas y tiempo de residencia de las aguas del sector "EL PINDO", Ensenada de Tumaco

\section{Corrientes}

La tabla 2 muestra los valores de velocidad de la corriente registrados a un metro de profundidad, en una estación fija ubicada en el lado occidental del puente, a través de un ciclo completo de marea, a partir de la máxima. Para eliminar los posibles efectos de factores hidrológicos (a saber, caudal del aporte fluvial), meteorológicos (vientos, evaporación, etc.), y/o antropogénicos (tráfico marítimo) sobre los registros de corriente, los datos presentados corresponden a promedios obtenidos a partir de observaciones a lo largo del período de estudio.

Puede notarse que durante el reflujo, la velocidad de la corriente en el punto de muestreo tiende a aumentar, siendo significativamente mayor que durante el flujo. En general, las velocidades máximas se observan entre 1-3 horas después de la pleamar y bajamar.
Tabla 2.

Velocidad de la corriente en una estación fija ubicada en el sector occidental del puente El Pindo (Ensenada de Tumaco) durante un ciclo completo de marea (nov/92 dic/92)

\begin{tabular}{|c|c|}
\hline TIEMPO (min) & VELOCIDAD m/seg \\
\hline 00:00 - 00:45 & $0.098+/-00334$ \\
$01: 00-01: 45$ & $0.103+/-0.057$ \\
$02: 00-02: 45$ & $0.101+/-0.056$ \\
$03: 00-03: 45$ & $0.100+/-0.041$ \\
$04: 00-04: 45$ & $0.103+/-0.027$ \\
$05: 00-05: 45$ & $0.091+/-0.036$ \\
$06: 00-06: 45$ & $0.073+/-0.025$ \\
$07: 00-07: 45$ & $0.040+/-0.007$ \\
$08: 00-08: 45$ & $0.045+/-0.005$ \\
$09: 00-09: 45$ & $0.043+/-0.011$ \\
$10: 00-10: 45$ & $0.035+/-0.011$ \\
$11: 00-11: 45$ & $0.058+/-0.019$ \\
$12: 00-12: 45$ & $0.085+/-0.005$ \\
\hline
\end{tabular}

Los resultados obtenidos para la dirección de corrientes superficiales de marea en las estaciones consideradas son presentados en las figuras 8 y 9 , donde pueden apreciarse los cambios en la dirección de la corriente tanto de flujo como de reflujo cerca del período de media marea. Ambas corrientes, se orientan preferencialmente según la dirección de los canales existentes; sin embargo, considerando la profundidad media del sector y la complejidad de la batimetría, el curso de la corriente superficial en cada uno de los subsectores parece estar condicionado por las características locales. 


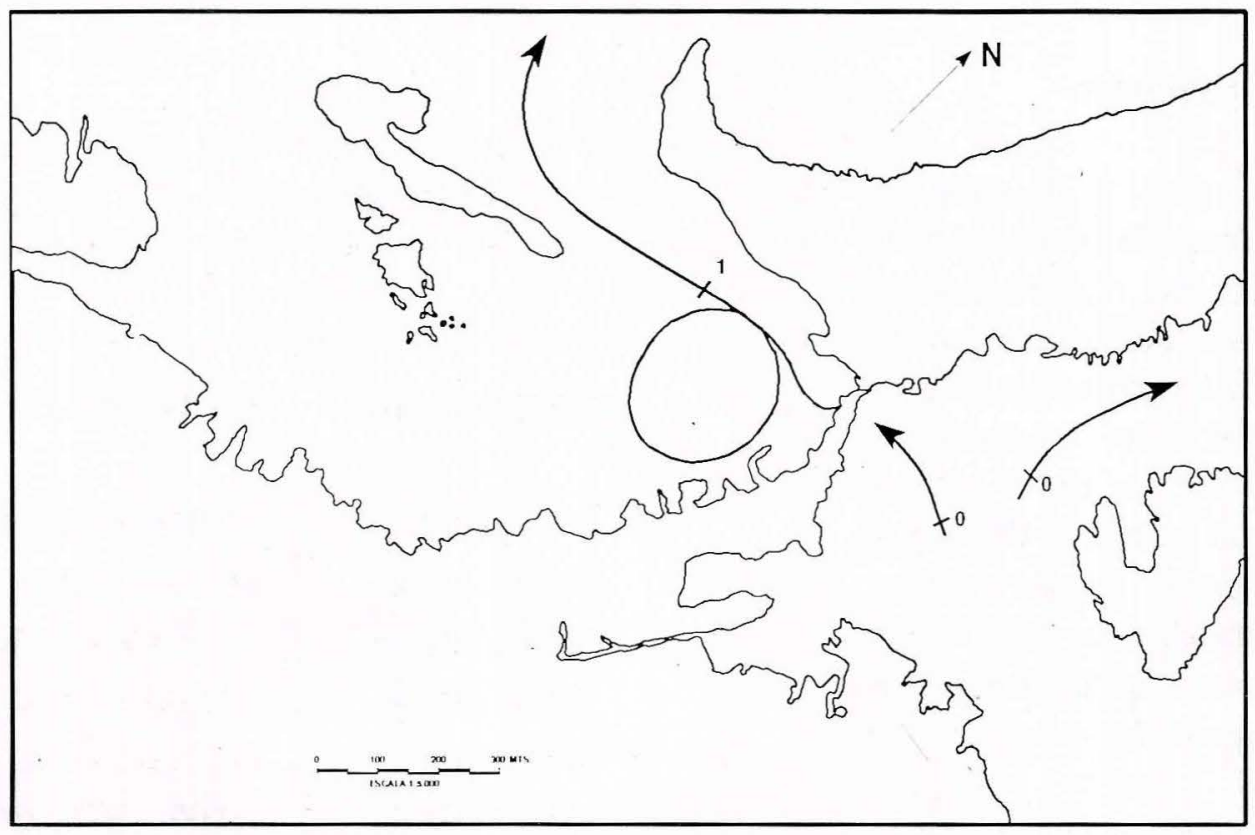

Figura 8

Dirección predominante de la corriente superficial durante la primera fase del período de reflujo

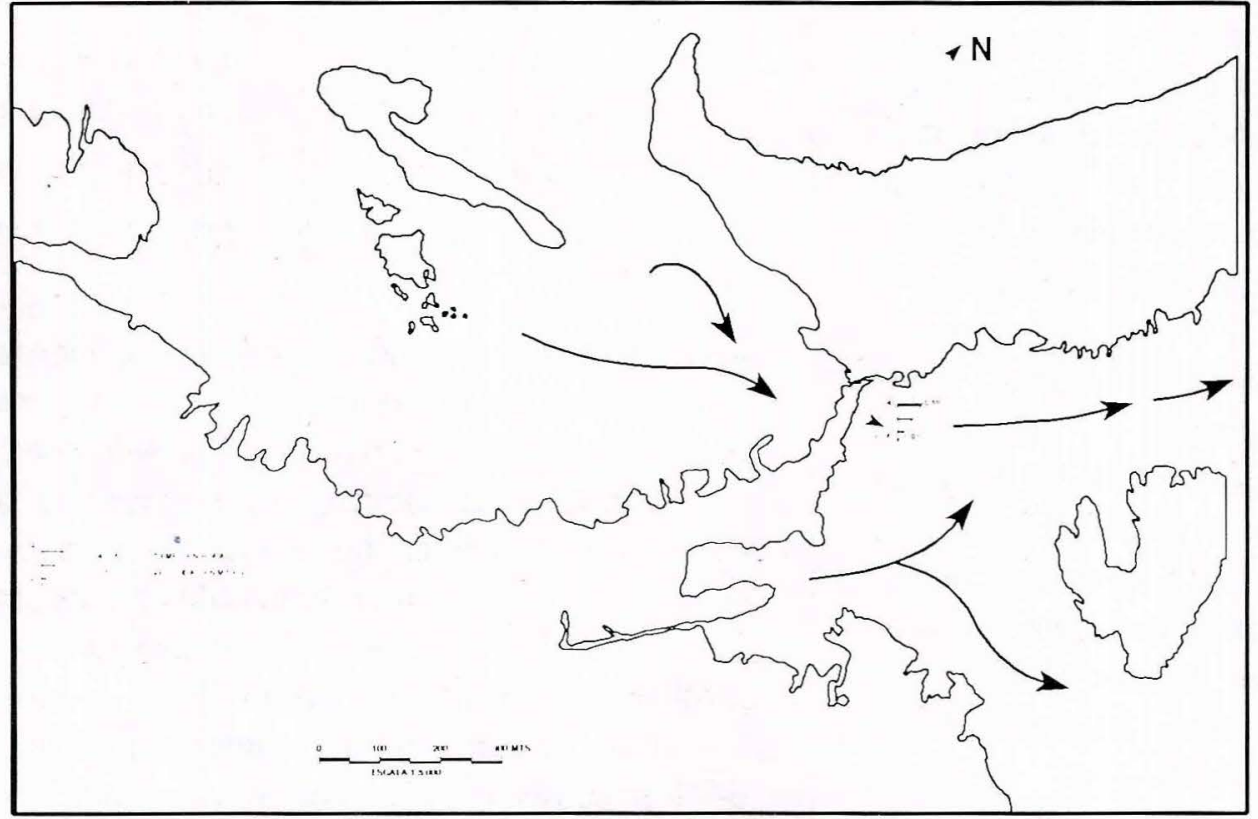

Figura 9

Dirección predominante de la corriente superficial durante la segunda fase del periodo de reflujo 
Con respecto al fondo, no se detectaron corrientes significativas. Se asume que las superficiales, con aguas de menor salinidad son las responsables del intercambio entre el sector y las aguas oceánicas circundantes.

\section{Aspectos Meteorológicos}

La tabla 3, reporta los valores promedios mensuales de algunos parámetros meteorológicos registrados durante 1992. Entre éstos y para efecto del análisis de los resultados oceanográficos obtenidos en el presente trabajo, son fundamentales la pluviosidad y los vientos. La pluviosidad, se considera importante porque se constituye en un factor de disminución de la salinidad superficial en el sector, bien sea por acción directa o por su efecto sobre el caudal de aguas dulces. Para el período de estudio, este parámetro mostró los valores más bajos en el año, pudiéndose catalogar el período noviembre- diciembre/92 como seco para la ensenada de Tumaco.

La velocidad y dirección del viento, son parámetros cuya influencia se manifiesta básicamente sobre el agua superficial. En comparación con los valores reportados a lo largo del año, los del período de estudio pueden considerarse como relativamente bajos. Observaciones durante este período y los datos de velocidad de corriente superficial, parecen indicar que el viento favorece o estimula la corriente de reflujo e inhibe la de flujo constituyéndose, junto con la configuración topográfica del fondo, en un factor que determina su curso.

\section{Modelo De Caja}

Una vez presentados los parámetros involucrados en el patrón hidrodinámico prevaleciente en el sistema en estudio y considerando que en el lado occidental del puente existe un movimiento neto de agua oceánica entrante en la capa inferior y agua de mezcla saliente en la superior, se deduce que existe una determinada profundidad entre estas dos capas donde el movimiento horizontal neto es cero, pero en la cual se da el transporte vertical de la capa inferior a la superior, sabiendo además que en el lado oriental hay una columna de agua con mayor mezcla, sin estratificación; en aras de expresar el proceso fisico del movimiento del agua en el sistema y poder determinar la intensidad del intercambio, se aplicó un modelo de caja, calculando los tiempos de residencia de acuerdo con lo propuesto por Schauss (1974) y Pilson (1985).

Para este caso y con base en lo expresado por Schauss (op. cit.), se dividió el sistema en dos grandes cajas: 


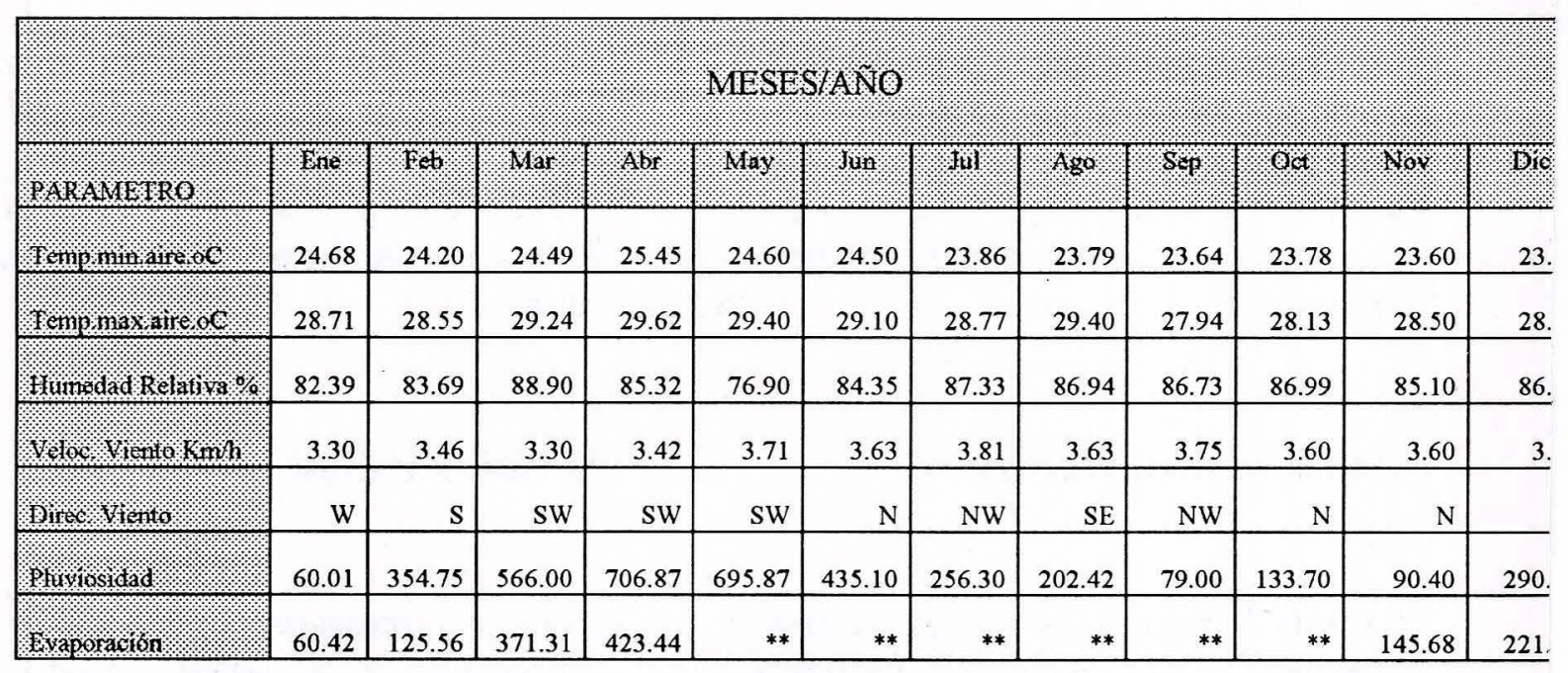

** Datos no disponibles

\section{Tabla 3}

Valores promedio mensuales de algunos parámetros meteorológicos reportados durante 1992, Ensenada de Tumaco.

Una, el lado occidental, que se trató como un estuario y la otra, el oriental, como una bahía. A continuación se describe el proceso aplicado en cada caso para el cálculo del período de renovación ó tiempo de residencia.

\section{Tiempo De Renovación De La Caja Occidental (Estuario)}

\section{Aplicando el método de Schauss}

Conociendo la longitud $(1=804 \mathrm{~m})$, el ancho $(\mathrm{a}=532 \mathrm{~m})$, la profundidad promedio $(\mathrm{z}=3 \mathrm{~m})$ y la profundidad de la capa superior $\left(z^{\prime}=1 \mathrm{~m}\right)$ del sistema, ésta última se establece mediante el análisis de los registros obtenidos a lo largo del presente estudio y por reportes de Mosquera (com. pers.), correspondientes a la capa de agua promedio donde se mezclan la fluvial con la oceánica y que coincide con la profundidad de la interfase correspondiente a aquella sin movimiento horizontal neto; asumiendo que este lado del sistema tiene una forma rectangular, se puede calcular fácilmente su área promedio (s), resultando: 
$\mathbf{s}=1 \times \mathrm{a}=804 \mathrm{~m} \times 532 \mathrm{~m}=427,718 \mathrm{~m} 2$

A partir de estos datos se llega al conocimiento de:

$$
\begin{aligned}
\mathbf{A}= & \begin{array}{l}
\text { Volumen del agua en la capa } \\
\text { superior a nivel medio }
\end{array} \\
\mathbf{A}= & s \times z^{\prime \prime}=427,718 \mathrm{~m} 2 \times 1 \mathrm{~m} \\
= & 427,718 \mathrm{~m} 3
\end{aligned}
$$

$$
\begin{aligned}
\mathbf{B}= & \begin{array}{l}
\text { Volumen de agua en la capa inferior } \\
\text { a nivel medio }
\end{array} \\
\mathbf{B}= & \mathrm{s} \times \mathrm{z}^{\prime \prime}=427,718 \mathrm{~m} 2 \times 2 \mathrm{~m} \\
= & 855,456 \mathrm{~m} 3
\end{aligned}
$$

donde,

$$
\mathbf{z}^{\prime \prime}=\text { Profundidad de la capa inferior }
$$

$$
\begin{aligned}
\mathbf{V}= & \text { Volumen total del estuario a nivel } \\
& \text { medio } \\
\mathbf{V}= & A+B=427,718 \mathrm{~m} 3+855,456 \mathrm{~m} 3 \\
= & 1 ' 283,184 \mathrm{~m} 3
\end{aligned}
$$

$$
\begin{aligned}
& \mathbf{P A}=\text { Porcentaje de A dentro del } \\
& \mathbf{P A}=100(\mathrm{~A} / \mathrm{V}) \\
& =100(427,718 \mathrm{~m} 3 / 1 ' 283,184 \mathrm{~m} 3) \\
& =33.3 \%
\end{aligned}
$$

$\begin{aligned} \mathbf{P B}= & \begin{array}{c}\text { Porcentaje de } B \text { dentro del } \\ \text { volumen total del estuario }\end{array} \\ \mathbf{P B}= & 100(\mathrm{~B} / \mathrm{V}) \\ = & 100(855,456 \mathrm{~m} 3 / 1 ' 283,184 \mathrm{~m} 3) \\ = & 66.6 \%\end{aligned}$

$\mathbf{R}=\quad$ Volumen de agua fluvial que entra al estuario durante un ciclo completo de la marea. Se calculó, considerando que el caudal promedio de agua que ingresó al estuario fué de 1.38 $\mathrm{m} 3 / \mathrm{seg}$. Por tanto, durante un ciclo de marea de 12 horas, $\mathbf{R}=\mathbf{5 9 , 6 1 6 ~ m 3 . ~}$

$\mathbf{Q A}=$ Volumen de agua que sale del estuario por la capa superior durante un ciclo completo de marea. El flujo promedio de agua que sale del estuario por la capa superior es $9.83 \mathrm{~m} 3 / \mathrm{seg}$. Por tanto, durante un ciclo de marea de 12 horas, $\mathbf{Q A}=$ $424,800 \mathrm{~m} 3$ 
$\mathbf{Q B}=$ Volumen de agua que entra al estuario por la capa inferior durante un ciclo completo de la marea. Como, $Q A=R+Q B$, resulta que: $Q B=365,184 \mathrm{m3}$

$\mathbf{r A}=$ Proporción de intercambio de agua en la capa superior sobre cada ciclo completo de la marea.

$\mathbf{r A}=\mathrm{QA} / \mathrm{A}=0.993$

$\mathbf{r B}=\quad$ Proporción de intercambio de agua en la capa inferior sobre cada ciclo completo de la marea.

$$
\mathbf{r B}=\mathrm{QB} / \mathrm{B}=0.427
$$

Si se considera que en un momento dado el estuario contiene sustancias que están uniformemente distribuidas (sólidos, nutrientes, etc.) de forma tal que la capa superior contenga $\mathbf{P A} \%$ y la inferior $\mathbf{P B} \%$, después del primer ciclo de marea, el porcentaje de estas sustancias que salió del estuario, está dado por rA x PA y el porcentaje que pasó de la capa inferior a la superior está dado por rB x PB siendo la concentración remanente en la capa superior después del primer ciclo de marea igual a:

$$
\mathbf{C A 1}=\mathrm{PA}(1-\mathrm{rA})+(\mathrm{rB} \times \mathrm{PB})
$$

y la que queda en la inferior:

$$
\mathrm{CB} 1=\mathrm{PB}(1-\mathrm{rB})
$$

Se puede calcular la concentración en ambas capas después de $\mathbf{n}$ ciclos de marea, pero debido a que en la medida que $\mathbf{n}$ aumenta, la ecuación se torna más compleja, Schauss (1974) propone el uso de una constante $r^{*}=\mathbf{1 / 2}(\mathbf{r A}+\mathbf{r B})$, que para el caso en estudio corresponde a 0.712. Con su aplicación, el porcentaje de los contaminantes originales que queda en el sistema después de $\mathbf{n}$ ciclos completos de la marea será:

$$
\mathrm{Cn}=\mathrm{CAn}+\mathrm{CBn}
$$

Para facilidad de cálculos, se recomienda el uso de logaritmos de tal modo que aplicando los términos:

$$
\begin{aligned}
& \mathbf{D}=\log (\mathrm{PA})+\operatorname{nlog}(1-\mathrm{rA}) \\
& \text { rB x PB } \\
& \mathbf{E}=\log (n)+\log \{----\}+\log \left(1-r^{*}\right) n \\
& \left(1-r^{*}\right)
\end{aligned}
$$


Entonces,

CAn $=\operatorname{alog}(\mathrm{D})+\operatorname{alog}(\mathrm{E})$

CBn $=\operatorname{alog}\{\log (\mathrm{PB})+\operatorname{nlog}(1-\mathrm{rB})\}$

Con estas ecuaciones, es posible conocer las variaciones del contenido de alguna sustancia en el sistema. La figura 10, presenta el comportamiento de los datos obtenidos del cálculo de la concentración, en función del tiempo, en las capas superior, inferior $y$ en el estuario en general.

Para efectos prácticos, se asume n como el número de días transcurridos.

Puede observarse, por este método, que al segundo día ya se ha eliminado más de la mitad de la concentración de la sustancia en la capa superior, siendo más lenta su eliminación en el fondo. Al cabo de siete días se observa que en la capa superficial sólo queda aproximadamente $3 \%$ del agua inicial.

Por lo tanto, el tiempo de renovación estimado para las aguas del sector occidental es de 2-5 días.

\section{Aplicando el método de Pilson}

De acuerdo con este método, el tiempo de residencia se evalúa como una función del ingreso de agua dulce.
El volumen de agua dulce promedio durante cada mes se calcula como:

$$
\mathrm{Vf}=\{1-(\mathrm{Sb} / \mathrm{So})\} \mathrm{Vb}
$$

donde,

$$
\begin{aligned}
\mathbf{V f} & =\text { Volumen de agua dulce } \\
\mathbf{V b} & =\text { Volumen del sistema } \\
& =1 ' 283,184 \mathrm{~m} 3
\end{aligned}
$$

$\mathbf{S b}=$ Salinidad media de la capa superior $=26.35 \mathrm{o} / 00$

So = Salinidad del agua oceánica en el fondo $=33.50 \mathrm{o} / \mathrm{oo}$

Entonces,

$$
\mathbf{V f}=273,874 \mathrm{~m} 3
$$

El tiempo tomado por el volumen total de agua dulce en el sistema para ser igualado por el agua que ingresa es:

$$
\mathbf{T}=\frac{\mathrm{Vf}}{\operatorname{Fw}(86,400)}=2.30 \text { días }
$$




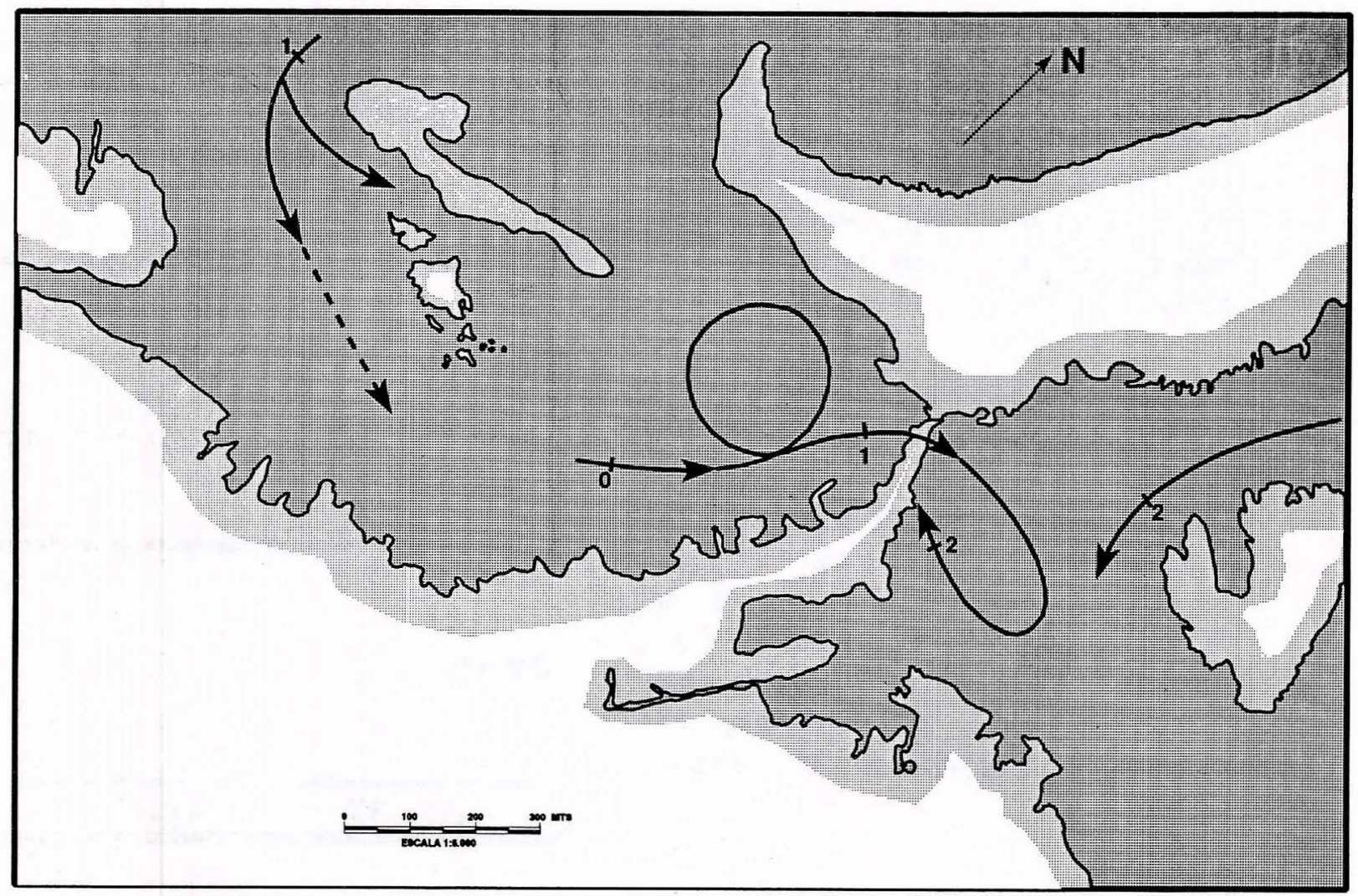

Figura 10

Dirección predominante de la corriente superficial durante la primera fase del periodo de flujo en el área de estudio, sector EL PINDO 
Un modelo de caja aplicado al transporte de partículas y tiempo de residencia de las aguas del sector "EL PINDO", Ensenada de Tumaco

donde,

$\mathbf{T}=$ Tiempo de renovación en días

$\mathbf{F w}=$ Ingreso promedio mensual de agua dulce $=1.38 \mathrm{~m} 3 / \mathrm{seg}$

Como puede observase, este valor está dentro del rango estimado por el método anterior.

\section{Tiempo De Renovación En La Caja Oriental (Bahía)}

Aprovechando el desarrollo teórico para estuarios, se puede simplificar el problema, despreciando la contribución fluvial R. De este modo, la bahía consta de una sóla capa de agua que se extiende desde la superficie hasta el fondo, pudiendo describirse con base en los siguientes parámetros:

V1 = Volumen de agua en el sistema considerando el Nivel Medio de las Mareas Altas (NMMA)

\section{V1 $=\mathrm{S} \times \mathrm{Z}=1297,990 \mathrm{~m} 3$}

$\mathbf{V} \mathbf{2}=$ Volumen de agua en el sistema acuerdo Nivel Medio de las Mareas Bajas (NMMB)

$$
\mathbf{V} 2=\mathrm{S}^{\prime} \mathrm{x} Z^{\prime}=585,093 \mathrm{~m} 3
$$

$\mathbf{P M}=$ Prisma de marea

$=V 1-V 2=712,897 \mathrm{~m} 3$

$\mathbf{r}=\quad$ Proporción de intercambio de agua entre el sistema y el mar sobre un ciclo completo de marea

$$
\mathbf{r}=\mathrm{PM} / \mathrm{V} 1=0.549
$$

De este modo, la concentración de una sustancia $\mathbf{C n}$ que queda en el sistema después de $\mathbf{n}$ ciclos de marea (días para el presente caso) está dada por:

$$
\mathbf{C n}=\operatorname{alog}\{\log (\mathrm{Co})+\operatorname{nlog}(1-r)\}
$$

donde,

\section{Co es la concentración inicial.}

La figura 11, presenta el comportamiento de dicha concentración en función del tiempo, para la estimación del tiempo de residencia en este sector, asumiendo como ejemplo un Co de 250 o/oo. A pesar de que el rango para el tiempo de residencia de las aguas en el sector oriental es muy similar al observado para el occidental, puede notarse que para el segundo día, el porcentaje de disminución de la concentración de aguas contaminadas es menor. 


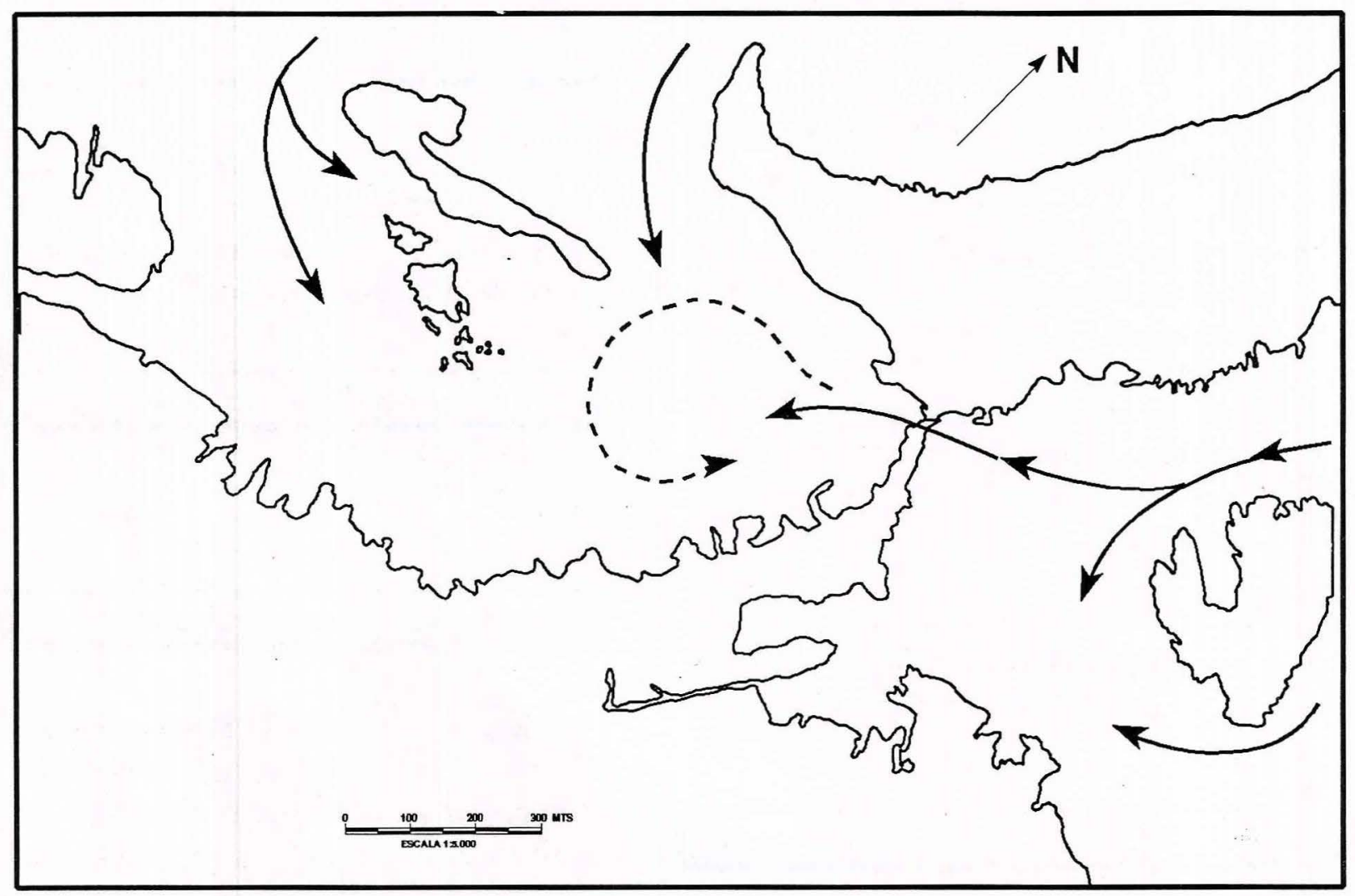

Figura 11

Dirección predominante de la corriente superficial durante la segunda fase del periodo de flujo en el área de estudio, sector LL PINDO 


\section{ISCUSION Y \\ C ONCLUSIONES}

De acuerdo con la definición de estuario de Pritchard (1973), el sector El Pindo puede tratarse como un estuario, debido al significativo aporte fluvial proveniente de las aguas de un brazo del río Mira que desemboca en el llamado "estero Guandarajo", al suroeste del sector de estudio y que, muy probablemente, es el responsable de la existencia de una capa superficial de menor salinidad.

En el medio marino, una de las áreas de mayor influencia del hombre como consecuencia del aprovechamiento de los recursus disponibles, son las zonas costeras y entre ellas, los estuarios.

Los mayores problemas de contaminación marina, a menudo, pueden atribuirse a la descarga de grandes volúmenes de desecho cuyo impacto en estas zonas, es local.

Los datos presentados en la Tabla 1 corroboran los reportes sobre el establecimiento progresivo de condiciones de baja calidad en las aguas de El Pindo (Anónimo, 1983; Mosquera, 1992; 1993).

Vale la pena resaltar que los altos valores de $\mathrm{DBO} 5$ prevalecientes en el área, pueden estar relacionados con la alta descomposición que se origina como consecuencia del vertimiento y acumulación de desechos de diferente tipo.

Algunos de estos desechos, pueden ser aportados por los ríos que además se constituyen en una fuente de agua dulce, promoviendo la presencia de una capa superficial de baja salinidad.

En el caso de El Pindo, sin embargo, resulta curioso que esta influencia fluvial, es más notable en marea alta.

Durante la marea baja, como lo expresan los resultados obtenidos, existe una columna de agua más homogénea, sin una estratificación propiamente dicha.

Es posible que esta diferencia obedezca a aspectos geomorfológicos y/o batimétricos que permiten el contacto de las aguas salinas con las del río, en la zona, sólo cuando existe un volumen tal que permite sobrepasar una "barrera geomorfológica", que puede entrar en funcionamiento en el período de baja marea, aislando estos dos cuerpos de agua.

De existir esta barrera, podría atribuirse su origen al resultado de procesos naturales o como consecuencia de los constantes vertimientos de material vegetal (aserrín), 
que se acumula en los extremos y paulatinamente es arrastrado por las corrientes a otros sitios del área .

La acción de esta barrera tambien puede ser determinada por el caudal de las aguas fluviales, en cuyo caso se aumentaría la penetración de éstas en períodos de creciente y disminuiría, como en la época de estudio, cuando éste es relativamente bajo.

Podría cumplirse lo expresado por Fraser y Wilcox (1981) y Fraser (1986) referente a que la mayor estratificación de salinidad, ocurre durante el período de mayor flujo de agua dulce, que para el caso en estudio, coincidiría con el de más alta marea.

Con base en la aplicación del modelo de caja de Enfield (1976) y Aguilera (1986), considerando únicamente la entrada de agua dulce por pluviosidad o pérdida por evaporación, se obtuvo que en el lado occidental del puente, los efectos de estos parámetros sobre la salinidad son realmente despreciables (pérdidas de aproximadamente $28.76 \mathrm{~cm} / \mathrm{mes} \quad \mathrm{y}$ ganancia de $36.24 \mathrm{~cm} / \mathrm{mes}$ ), en comparación con el lado oriental donde éstas son de aproximadamente 575.12 $\mathrm{cm} / \mathrm{mes}$ y $724.88 \mathrm{~cm} / \mathrm{mes}$, respectivamente

Estos ingresos y salidas por efectos meteorológicos, inducen movimientos verticales de masas de agua que, a su vez, pueden influir sobre la distribución de la temperatura de tal modo que este parámetro, que no mostró una marcada estratificación a lo largo de la columna de agua, parece estar influenciado por factores ambientales los cuales, sumados a las corrientes oceánicas involucradas y a las características batimétricas del sector, le confieren altos valores.

De otro lado, la cantidad de agua dulce recibida de las zonas de drenaje y su grado de mezcla con agua salada resultan ser de fundamental importancia en la circulación vertical del sistema y pueden ser responsables de los cambios sufridos en la intensidad y dirección de la corriente de marea; según lo expresado por SHN y SOHMA (1989) referente a que la intrusión de agua salada en ambientes estuarinos se constituye en un factor importante en la hidrodinámica de éstos por ser un modificador de su régimen de flujo.

En el área de estudio, adicional a las corrientes de marea, otro factor de gran importancia en la hidrodinámica del sistema, es la profundidad.

A pesar de que éste presenta tres bocas para el drenaje, como lo muestran los vectores descritos para la velocidad de la corriente, sólo cuando la marea alcanza un determinado nivel, éstas se vuelven funcionales, dando como resultado un patrón de circulación distinto antes $\mathrm{y}$ 
después del nivel de media marea, tanto en el flujo como en el reflujo.

Del mismo modo, puede atribuirse a la topografia del fondo, la baja velocidad de la corriente en las aguas inferiores, debido a la existencia de sitios donde éstas se estancan, formando pequeñas y puntuales "lagunas" con poco o ningún intercambio.

Esto concuerda con lo expresado por Schauss (1974), quien señala que estas aguas inferiores no se consideran activas en el proceso de intercambio debido a que su circulación está restringida por la batimetría.

Todos estos factores, en conjunto, determinan la permanencia $y / o$ salida de masas de agua del sistema. Según Takeoka (1990), un sistema encerrado puede considerarse como un cuerpo de agua en el cual el flujo de material que entra y sale (Fo), está relacionado con la masa que permanece (Mo) mediante la razón To $=$ $\mathrm{Mo} / \mathrm{Fo}$, la cual tiene una dimensión de tiempo llamada "tiempo de residencia", llamada también "tiempo de renovación" ó "tiempo de tránsito promedio".

Los resultados obtenidos en el presente estudio muestran que, contrario a lo esperado, el manifiesto deterioro en la calidad de las aguas de El Pindo, posiblemente, no obedece a factores hidrodinámicos ó de circulación de sus aguas, como lo demuestra el bajo tiempo de residencia estimado ( 2 - 5 días).

Muy probablemente estas condiciones están relacionadas con la baja profundidad y con la calidad de los sedimentos de fondo, que a través de la interfase agua-sedimento, disminuyen la calidad de las aguas renovadas contribuyendo a la persistencia de las condiciones detectadas en ellas.

Las figuras 10 y 11 , muestran que en ambos subsectores los tiempos de residencia son muy similares.

Sin embargo, centrando la atención en el sector occidental, donde se detectaron las mayores anomalías bioquímicas, previo conocimiento de los tiempos de residencia estimados, podría pensarse que una solución a estos problemas sería la ampliación de la boca del puente.

Los resultados obtenidos, sin embargo, muestran que tal solución está más ligada con planeamiento de actividades de dragado de los sedimentos de fondo, dando especial atención a la selección del sitio de disposición de este material para evitar la extensión del problema a otras áreas en proximidad o dentro de la ensenada.

Con este dragado, además de eliminar sedimentos en un alto grado de descomposición, generadores de gases 
(sulfuro de hidrógeno y metano), nutrientes, altos niveles de materia orgánica y malos olores, se estará garantizando una mayor circulación y renovación de las aguas, al romperse las barreras existentes tanto para el flujo de agua dulce como salada.

De otro lado, mejorarán las condiciones operativas del sector permitiendo el tránsito de embarcaciones en los diferentes niveles de la marea.

\section{R ECOMENDACIONES}

Para resolver el problema detectado en el sector El Pindo, considerado como un foco potencial de eutroficación en la ensenada de Tumaco y teniendo en cuenta que de acuerdo con los tiempos de residencia estimados para sus aguas, el problema no obedece a factores hidrodinámicos, se recomienda planear actividades de dragado, previo estudio de impacto ambiental, para remoción de los sedimentos de fondo.

Por otro lado, considerando que la baja calidad de las aguas es producto de la descomposición de materiales acumulados en el fondo, en este sector, se recomienda suspender la descarga de los desechos sólidos provenientes del aprovechamiento de la madera en los aserríos de la zona y el establecimiento de medidas de control efectivas por parte de las autoridades regionales competentes.

Se considera que los resultados obtenidos para este sector, pueden aplicarse a algunas áreas circundantes muy cercanas $\mathrm{y}$, muy especialmente, tenerse en cuenta en caso de un eventual derrame de sustancias como los hidrocarburos del petróleo, que representan un riesgo potencial, debido a que la tubería del oleoducto atraviesa este sector desde el terminal hasta el sitio de descargue de petroleros a aproximadamente 2 millas de la costa. 


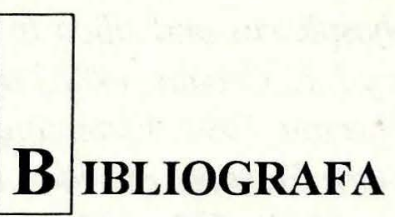

AGUILERA, J. Clasificación del estuario antiguo cauce del río Sinú. Tesis de grado. Facultad Oceanografía Física. Escuela Naval Almirante Padilla. Armada Nacional., 1986, 108 p.

ANÓNIMO.. Niveles actuales del oxígeno disuelto y salinidad en la bahía interna de Tumaco. Centro Control Contaminación del Pacífico. 1983, PP45

APHA. Standard methods for the examination of water and waste water. Edición 15. APHA/AWWA/WPCF.1981, 1134 p.

CONOMOS, T.J. Properties and circulation of San Francisco Bay waters. Am. Assoc. Adv. Sci. 1979, Pág. 47 - 84.

CORREA, I.D., J.L. GONZALEZ y RODRIGUEZ. C.A.. Geomorfología general y sedimentología de la bahía de Tumaco. Memorias VI Seminario Nacional Ciencias del Mar Comisión Colombiana de Oceanografía - Universidad Jorge Tadeo Lozano. Pág 233 - 239. Enfield, D. 1976. Práctica No. 8, Modelos de caja en Mamual de prácticas de oceanografía fisica. INOCAR. Pág 67 - 75.

ENTREVISTA con Alba Idalia Mosquera. Jefe de la Sección de Contaminación, Centro Control Contaminación del Pacífico, Tumaco, 25 nov 1992. 
FRASER, T.H. y. WILCOX. W.H . Enrichment of a subtropical estuary with nitrogen, phosphorus and silica in Estuaries and mitrients editado por B.J. Neilson y L.E. Cronin. 1981.Pág 481 - 498. Citado por Miller, R.L. y B.F. Mc Pherson. 1991. Estimatıng estuarine flushing and residence times in Charlotte Harbor, Florida via salt balance and box model. Limnol. Oceanogr. 36 (3): 602 - 612.

FRASER, T.H. Long term water quality Characteristics of Charlotte Harbor. US. Geol. Sur. Water-Resour. Invest. Rep. 1986, Pág. 86 - 110. Citado por Miller, R.L. y B.F. Mc Pherson. 1991. Estimating estuarine flushing and residence times in Charlotte Harbor, Florida via salt balance and box model. Limnol. Oceanogr. 36 (3): 602 - 612.

GOODWIN, C.R. . Tidal flow, circulation and flushing changes caused by dredge and fill in Tampa Bay, Florida. US. Geol. Surv. Water Supply 1987, 2282 p. Citado por Miller, R.L. y B.F. Mc Pherson. 1991. Estimating estuarine flushing and residence times in Charlotte Harbor, Florida via salt balance and box model. Limnol. Oceanogr. 36 (3): 602 612.

MARRUGO, A.J. Estudio de la contaminación marina por hidrocarburos en el litoral pacífico colombiano. Boletín Científico CCCP (1): 41 - 54.----- 1991. Estudio de la Contaminación marina por hidrocarburos en el litoral pacífico colombiano 1989-1990. Boletin Científico CCCP (2): 3 - 32

MILLER, R.L. y. MCPHERSON. B.F . Estimating estuarine flushing and residence times in Charlotte Harbor, Florida via salt balance and box model. Limnol. Oceanogr. 1991, 36 (3): 602 - 612.

MOSQUERA, A. I. . La Contaminación orgánica, un posible precursor de la eutroficación en aguas de la Ensenada de Tumaco. Agosto/90 Agosto/91. Boletín Científico CCCP (3): 31 - 50 ---1993. Estado actual de la eutroficación en aguas costeras de la Ensenada de Tumaco y diagnóstico en la Bahía de Buenaventura. Boletín Cientifico CCCP 1992, (4): $17-26$ 
OFFICER, C.B. . Box model revisited. En Estuarine and Wetland processes with emphasis on modeling plenum editado por P. Hamilton y K. E. Mac Donald. 1980, Pág. 65 - 114.

PILSON, M.E.Q. . On the residence time of water in Narraganset Bay. Estuaries 8: 2 - 14, 1985.

PRITCHARD, D.E. . Salt balance and exchange rate for Chiconteague Bay. Chesapeake Sci. 1: 48 - 57. ----- 1973. La circulación y la Mezcla en las regiones costeras y los estuarios en Oceanografía, la última frontera compilado por R.C. Vetter. Editorial El Ateneo. Capitulo 26: $273-282,1960$.

SCHAUSS, R.H. La estimación del período de renovación de las aguas de un estuario o bahía con aplicación a la bahía de Cartagena de Indias. Ministerio de Defensa. Armada Nacional. Dirección General Maritima. 1974, DO (21): 26 p.

SHN y SOHMA. . Estudio para la evaluación de la contaminación en el Rio de La Plata. Informe de Avance Comisión Administrativa del Rio de La Plata.(422). 1989, $423 \mathrm{p}$.

STRICKLAND, J.D.H. y PARSONS, T.R. . A practical handbook of sea water analysis. Fish. Res. Board of Canada. Segunda edición. Otawa. 1972

TAKEOKA, H. Fundamental concepts of exchange and transport time scales in a coastal sea. Cont. Shelf. Res. 3: 311 - 326. - --- 1990. Water exchange and transport of matter in the Seto Inland sea. Marine Pollution. 1984, 23: 41 - 44.

WALTERS, R.A; CHENG R.T y CONOMOS, T.J. . Time scales of circulation and mixing processes of San Francisco Bay waters. Hidrobiología 1985, 129: 13 - 36. 